\title{
Sériation des adjectifs dans le SN et formation de concepts
}

Denis Bouchard

\section{(2) OpenEdition \\ Journals}

Édition électronique

URL : http://journals.openedition.org/rlv/1383

DOI : $10.4000 /$ rlv. 1383

ISSN : 1958-9239

Éditeur

Presses universitaires de Vincennes

\section{Édition imprimée}

Date de publication : 1 décembre 2005

Pagination : 125-142

ISBN : 2-84292-176-3

ISSN : 0986-6124

\section{Référence électronique}

Denis Bouchard, «Sériation des adjectifs dans le SN et formation de concepts », Recherches linguistiques de Vincennes [En ligne], 34 | 2005, mis en ligne le 22 décembre 2006, consulté le 19 avril 2019. URL : http://journals.openedition.org/rlv/1383; DOI : 10.4000/rlv.1383 


\title{
Denis BOUCHARD
}

Université du Québec à Montréal

\section{SÉRIATION DES ADJECTIFS DANS LE SN ET FORMATION DE CONCEPTS ${ }^{1}$}

\begin{abstract}
RÉSUMÉ
En français, il y a un ordre préféré pour les adjectifs lorsqu'ils sont du même côté du Nom à l'intérieur du syntagme nominal. Ces adjectifs sont interprétés dans un emboîtement : l'adjectif immédiatement juxtaposé au nom modifie ce nom, l'adjectif juxtaposé à ce groupe le modifie, et ainsi de suite. Les sériations dépendent du sens des adjectifs et du contexte d'emploi : l'adjectif qui est le plus susceptible de former un concept avec le nom selon la situation d'emploi est celui qui se combine immédiatement avec le nom, qui est le plus enchâssé. Les sériations ne représentent que des préférences et non pas des contraintes rigides, et il est presque toujours possible d'intervertir l'ordre des adjectifs. Les combinaisons résultantes ne constituent pas des déviances syntaxiques, mais plutôt des combinaisons sémantiques moins attendues : les expressions sont acceptables si le nom et l'adjectif adjacent construisent un concept provisoire qui est approprié dans le contexte.
\end{abstract}

\section{MOTS-CLÉS}

Adjectifs, ordre, concepts provisoires. 


\section{La sériation des adjectifs en français et en anglais}

Il y a un certain ordre préféré pour les adjectifs lorsqu'ils sont du même côté du Nom à l'intérieur du syntagme nominal, que ce soit en position postnominale comme en (1) ou en position prénominale comme en (2) et (3).

(1) a. une situation financière désastreuse ?une situation désastreuse financière

b. des mesures techniques brutales ?des mesures brutales techniques

c. une réaction violente immédiate aux actions de Washington ?une réaction immédiate violente aux actions de Washington
a. a disastrous financial situation
?a financial disastrous situation
b. brutal technical measures
?technical brutal measures
c. an immmediate violent reaction to the actions of Washington
?a violent immmediate reaction to the actions of Washington
a. un beau petit chien
\#un petit beau chien
a'. a nice small dog
\#a small nice dog
b. ce supposé nouveau miracle
?ce nouveau supposé miracle
b'. that alleged new miracle
?that new alleged miracle

Comme on le constate en comparant les exemples correspondants du français et de l'anglais, l'anglais n'accepte généralement les adjectifs sans complément qu'en position prénominale, alors que le français les accepte tant devant qu'après le nom. De plus, l'ordre des adjectifs prénominaux est l'inverse de celui des adjectifs postnominaux: ainsi on a brutal technical measures comparé à des mesures techniques brutales. Nous ne traiterons pas ici du problème de la variation linguistique, ni non plus des facteurs qui favorisent une position prénominale ou postnominale de l'adjectif en français, ni des différences de sens selon que l'adjectif est dans l'une ou l'autre de ces positions, puisque nous en avons déjà traité en détail dans Bouchard (2002). Nous examinons donc uniquement les facteurs qui déterminent la sériation des adjectifs.

Les adjectifs apparaissent dans un ordre préféré qui dépend de leur sens. Les auteurs divergent un peu sur les détails de cet ordre idéal, mais on retrouve certains éléments communs dans toutes les descriptions. Ainsi, pour l'anglais, Leech \& Svartvik (1975) proposent l'ordre en (4).

\section{(4) Opinion $>$ Taille $>$ Âge $>$ Forme $>$ Couleur $>$ Origine $>$ Matériel}

De nombreux autres auteurs ont proposé de telles sériations, avec quelques divergences mineures (voir Sproat \& Shih 1988 pour un aperçu de plusieurs de ces suggestions). Comme plusieurs l'ont fait remarquer, de telles sériations des adjectifs ne représentent que des préférences et non pas des contraintes absolues. Il est presque toujours possible d'intervertir l'ordre des adjectifs. Le changement de l'ordre entraîne un changement de sens. Ceci est dû au fait que 
deux adjectifs du même côté du nom sont interprétés dans un emboîtement: l'adjectif immédiatement juxtaposé au nom modifie ce nom, alors que l'autre adjectif modifie le groupe nom + adjectif. Grevisse (1986 : 505) donne l'exemple des soins médicaux gratuits, où gratuits sert d'épithète à soins médicaux ${ }^{2}$. Une inversion de l'ordre des adjectifs produit un emboîtement différent, et donc une composition de sens différente. Les points d'interrogations en (1)-(3) ne représentent donc pas des déviances syntaxiques, mais plutôt des combinaisons sémantiques moins attendues; cependant, en construisant un contexte pragmatique approprié, on constate que ces expressions sont acceptables. Par exemple, dans un contexte où l'on discute de différents types de situations désastreuses, on peut comparer les maux et les remèdes pour une situation désastreuse financière, une situation désastreuse politique et une situation désastreuse familiale.

\section{Les analyses antérieures}

La structure minimale correspondant à l'emboîtement des adjectifs est comme en (5):
a. une [[situation financière] désastreuse]
b. un [beau [petit chien]]
c. un [[[métro aérien] suspendu] français]

Cette observation amène tous les auteurs à proposer un principe général d'analyse qui fait dépendre la sériation de l'interprétation sémantique: l'adjectif dont la sémantique se prête le mieux à une modification directe du nom tend à se retrouver plus près de ce dernier. Ainsi, en $(5 \mathrm{c})$, le sens de aérien est plus directement compatible avec le sens de métro que ne l'est celui de suspendu ou de français; de même, le sens de suspendu est plus directement compatible avec le sens de métro aérien que ne l'est celui de français. Mais ceci n'est qu'une tendance et un autre emboîtement peut être plus pertinent dans un contexte particulier. Par exemple, Forsgren (1978 : 189) pose qu'un adjectif relationnel comme européenne porte normalement directement sur le nom, comme en (6a), mais constate du même coup que l'ordre "logique" peut être inversé comme en (6b), où l'adjectif relationnel n'est plus le plus près du nom.

$$
\begin{aligned}
& \text { a. une solution européenne commune } \\
& \text { b. une solution commune européenne }
\end{aligned}
$$

Plusieurs auteurs vont au-delà d'un tel principe général d'analyse et tentent de rendre compte du détail de chacune des positions dans la sériation. Il y a trois types d'analyse des restrictions sur l'ordre des adjectifs: les analyses basées sur un patron, les analyses syntaxiques et les analyses sémantico-conceptuelles. 


\subsection{Les analyses basées sur un patron}

Les analyses du premier type proposent un patron comme celui en (4), où l'on donne la liste ordonnée de catégories sémantiques d'adjectifs. Dans les meilleurs des cas, ces listes décrivent assez bien l'ordre des adjectifs. Mais comme toute liste, elle ne font que décrire sommairement et n'expliquent rien. Les listes ne sont que des assertions d'existence: ce sont des inventaires de faits, elles nous disent ce qu'il y a, alors que la science vise beaucoup plus, elle s'intéresse aux modalités d'existence, à ce qu'il peut y avoir. On peut bien sûr penser qu'il n'y a rien d'intéressant ou de profond à expliquer à propos de l'ordre des adjectifs, qu'il est tel qu'il est et qu'on ne peut que spécifier les tendances de sériation. Mais nous verrons à la section 3 que ce n'est pas le cas, que l'ordre de combinaison des adjectifs est directement relié à la capacité de chaque adjectif de former une Sorte naturelle avec le nom, au sens de Carlson (1980 : 26sv.), Krifka et al. (1995) et Chierchia (1998).

\subsection{Les analyses syntaxiques}

Les analyses du deuxième type sont syntaxiques. Par exemple, au début de la grammaire générative, des analyses comme celle de Vendler (1968) comportaient des règles syntaxiques qui transforment des phrases relatives en syntagmes adjectivaux. L'ordre de surface des adjectifs dépend de l'ordre d'application des transformations. Ainsi, chez Vendler, les adjectifs appartiennent à différentes classes selon la paraphrase en phrase relative à laquelle ils correspondent, et une hiérarchie des classes détermine l'ordre dans lequel les transformations s'appliquent. Ce type d'analyse a donc une valeur explicative aussi faible que les analyses basées sur un patron: les éléments clés de l'analyse sont des listes ordonnées, de transformations dans ce cas-ci.

Les analyses syntaxiques de la sériation ont connu un renouveau dans les années 1990. On en trouve un exemple typique dans l'étude de Cinque (1994). (Voir aussi Abney, 1987; Valois, 1990, 1991; Bernstein, 1993, entre autres. Cinque 1999 étend cette approche à la distribution des adverbes.) Cinque pose que les adjectifs sont générés dans la position de spécifieur de catégories fonctionnelles qui correspondent à différentes classes sémantiques d'adjectifs. Pour rendre compte des sériations qu'il observe, données ici en (7), il propose qu'une catégorie fonctionnelle $\mathrm{F}^{\mathrm{n}+1}$ apparaissant à la droite d'une catégorie fonctionnelle $\mathrm{F}^{\mathrm{n}}$ est un complément sélectionné sémantiquement par $\mathrm{F}^{\mathrm{n}}$.

(7) Sériation des adjectifs dans les nominaux dénotant des objets: poss $>$ cardinal $>$ ordinal $>$ qualité $>$ taille $>$ forme $>$ couleur $>$ nation

Par exemple, la tête d'un syntagme Taille sélectionnerait un syntagme Forme, dont la tête sélectionnerait un syntagme Couleur, et ainsi de suite. Les 
éléments clés de l'analyse sont donc ici aussi des listes ordonnées, comme dans les analyses basées sur un patron, sauf que l'ordonnancement se fait par enchâssement cette fois-ci. De plus, cette analyse nécessite qu'on indique pour chaque adjectif à quelles sous-classes sémantiques il appartient, afin de déterminer les catégories fonctionnelles dont cet adjectif peut être le spécifieur; elle nécessite aussi des catégories fonctionnelles redondantes, puisqu'elles reproduisent exactement les sous-classes sémantiques d'adjectifs.

Ce nouveau cru d'analyses syntaxiques prétend aussi à l'universalité. Ainsi, l'enchâssement des catégories fonctionnelles qui produit les sériations en (7) serait le même pour toutes les langues. Cette conception syntaxique de l'universalité est très courante en grammaire générative et s'appuie sur l'intuition qu'il y a une position naturelle d'interprétation pour chaque élément, que certaines relations sémantiques s'expriment universellement par les positions relatives que les éléments occupent dans la phrase (Chomsky (2000) l'énonce très clairement). Cette position naturelle d'interprétation se trouve généralement à correspondre à une position de surface de l'anglais, qui, bien sûr, est conceptuellement très naturelle pour les théoriciens surtout anglophones de la grammaire générative ${ }^{3}$. Mais il faut alors expliquer pourquoi on trouve fréquemment des éléments dans des positions contre nature, comme les adjectifs dans l'exemple français en (8b), comparé à l'anglais (8a).

a. blue Chinese vases

b. vases chinois bleus

Cinque (1994) suggère que le français a la même liste universelle de catégories fonctionnelles que l'anglais, dans le même ordre fixe d'enchâssement devant le nom. Mais le français aurait en plus une liste de traits ininterprétables qui déclencheraient le déplacement de certains constituants. Ainsi, pour obtenir (8b), la structure de départ serait celle en (9a), identique à celle du syntagme anglais correspondant, sauf qu'elle comporterait un trait ininterprétable qui provoque le déplacement de vases vers une catégorie fonctionnelle devant chinois comme en (9b).
a. bleus chinois [vases] $==>$
b. bleus [vases] chinois $==>$
c. [[vases] chinois] bleus

Pour rendre compte du fait que les deux adjectifs sont postnominaux ici en français, et qu'ils apparaissent dans l'ordre inverse de celui prédit par la liste universelle de catégories fonctionnelles, on pose que le français a un autre trait additionnel, qui a pour effet que vases chinois est déplacé vers une autre catégorie fonctionnelle devant bleus, comme en (9c). La différence entre 
l'anglais et le français quant à la sériation des adjectifs en (8) est donc de nouveau attribuée à des listes: le français aurait des traits ininterprétables qui seraient absents de la liste de traits de l'anglais. Ces listes ne font que répéter qu'il y a des différences d'ordre entre les deux langues, sans rien expliquer ${ }^{4}$. En fait, une telle liste de catégories ordonnées par enchâssement équivaut à réintroduire dans la théorie une composante aussi excessivement puissante que des règles de réécriture. De plus, l'analyse perd complètement l'observation très ancienne que deux adjectifs postnominaux sont interprétés dans un emboîtement, tout comme deux adjectifs prénominaux. Ce n'est qu'après une série d'opérations tortueuses qu'on arrive à un ordre de surface qui se trouve à reproduire exactement l'ordre de composition sémantique, qui s'exprime pourtant très facilement: l'adjectif immédiatement juxtaposé au nom modifie ce nom, alors que l'autre adjectif modifie le groupe nom + adjectif.

\subsection{Les analyses sémantico-conceptuelles}

Tant les analyses à patron que les analyses syntaxiques font fortement emploi de notions sémantico-conceptuelles pour leurs catégorisations. De plus, les restrictions sur la sériation en termes de distance du nom sont très répandues, voire peut-être universelles. Ceci a amené plusieurs chercheurs à proposer une explication sémantico-conceptuelle de la sériation des adjectifs. Ainsi, Whorf (1945) propose que les adjectifs qui dénotent des qualités inhérentes (couleur, matériel, nationalité) se placent plus près du nom que les adjectifs dénotant des propriétés non inhérentes (forme, taille, position).

Poursuivant dans cette veine, Martin (1969 a, b) suggère que ce type de contrainte sur la sériation découle d'une stratégie de traitement cognitif. Il fait l'hypothèse que les syntagmes se construisent à partir de la tête et en s'éloignant vers la périphérie. Il suppose aussi que certaines propriétés sont plus apparentes que d'autres, et donc qu'elles sont plus saillantes, plus accessibles, et qu'elles tendent à être choisies avant les moins apparentes. En conséquence, les adjectifs exprimant des propriétés apparentes sont plus près du nom dans la sériation. Une propriété apparente est généralement plus absolue, en ce sens qu'elle exige moins de comparaisons ${ }^{5}$. Ainsi, affirme Martin, aucune comparaison n'est requise pour déterminer si un objet est noir, mais on a besoin d'une comparaison pour des propriétés de taille comme GRAND ou PETIT, d'où l'ordre large/small black shoe. Sproat \& Shih (1988) reprennent le même argument à propos de l'exemple large red car. D'après eux, déterminer si une voiture est grande exige un certain nombre de calculs: il faut établir de quel type d'objet il s'agit, et ensuite déterminer par comparaison s'il est grand pour ce type d'objet. Par contre, on peut déterminer qu'une voiture est rouge si une partie suffisamment grande de sa surface est 
rouge, ce qui impliquerait moins de calculs. Mais est-il si évident que les calculs pour déterminer que cette surface rouge est suffisamment grande sont plus simples que ceux pour déterminer que la voiture est grande? Comme je le fais remarquer dans Bouchard (2002: 120), on ne nous donne aucun critère permettant d'évaluer les deux types de calculs: tout ceci relève d'intuitions simplistes et semble entièrement a posteriori. De plus, la notion d'apparence relative des propriétés pose un problème empirique, du moins telle qu'elle est présentée. Ainsi, il semble que l'évaluation de la couleur exige autant de calculs que celle de la taille: la couleur aussi est calibrée en fonction du type d'objet dénoté par le nom. Un adjectif de couleur comme red/rouge exprime une propriété tout aussi relative qu'un adjectif de taille comme large/grand, tant quantitativement que qualitativement. Pour déterminer si chacun des éléments en (10) est considéré comme étant rouge ou non, il faut tenir compte du type d'objet dont il s'agit, de la partie de l'objet qui est rouge, de l'intensité de la couleur, etc.
a. Jean est rouge.
b. La voiture est rouge.
c. Elle boit du vin rouge.
d. Voilà une belle fraise rouge.

Par exemple, une fraise de la couleur du visage de Jean ne serait probablement pas considérée comme étant rouge.

Autre problème, on note aussi que l'équivalent français de l'exemple de Martin, une petite chaussure noire, pose problème: les deux adjectifs sont adjacents au nom, et il faudrait un critère pour déterminer lequel compte comme étant plus près du nom. La tâche se complique du fait que la "portée" des adjectifs est souvent ambiguë dans ces séquences: ainsi, une nouvelle proposition intéressante peut s'interpréter soit avec nouvelle qui a portée sur intéressante (une proposition intéressante qui s'ajoute aux autres propositions intéressantes) ou l'inverse (une nouvelle proposition qui a la propriété d'être intéressante).

Mumoratsu (2001) tente aussi de donner un fondement cognitif à la sériation des adjectifs. Elle part de l'observation de Bunt (1979) que certains adjectifs (dits comptables) comme small ne peuvent se combiner à des noms de masse (\#small metal vs small ball) ${ }^{6}$ et que d'autres adjectifs (dits de masse) comme white se combinent aussi bien avec des noms de masse que des noms comptables (white metal vs white ball). Elle suggère une tripartition des noms qui serait à la fois sémantico-conceptuelle et syntaxique: les noms abstraits seraient conceptuellement unidimensionnels (1D) avec une structure syntaxique simple; les noms de masse seraient 2D avec une structure syntaxique comportant un niveau fonctionnel de plus au-dessus de la structure des nom 1D; finalement, les noms comptables seraient $3 \mathrm{D}$ avec une structure 
syntaxique comportant un troisième niveau fonctionnel dans lequel la structure des nom 2D serait enchâssée. La complexité conceptuelle se reflèterait donc dans la complexité syntaxique. Un adjectif ne peut s'adjoindre qu'à une projection syntaxique de dimensionalité appropriée. Un adjectif de Taille comme small modifie une expression qui possède une forme, donc un niveau 3D. Par contre, de tels adjectifs comptables ne peuvent s'adjoindre à la structure des noms de masse car ces noms n'ont pas le niveau supérieur 3D requis par ces adjectifs. Par contre, comme la projection fonctionnelle 3D (comptable) domine la projection 2D (masse), les adjectifs de masse peuvent s'adjoindre à ce niveau des noms comptables, d'où white ball. De plus, ceci prédit que les adjectifs comptables doivent précéder les adjectifs de masse, puisque Mumoratsu adopte l'hypothèse que les adjectifs sont adjoints à la gauche de la projection fonctionnelle de dimensionalité appropriée ${ }^{7}$.

L'analyse de Mumoratsu semble simple, mais elle comporte plusieurs hypothèses sous-jacentes qui la minent. Par exemple, selon l'hypothèse que le Comptable domine la Masse, et que la Masse domine l'Abstrait, un nom comptable serait décomposable et comprendrait un élément de Masse et un élément d'Abstrait. Quel sens doit-on donner à cette affirmation, et est-ce que cela repose sur une base cognitive valable? Et même si c'était le cas, est-ce que cela implique que l'enchâssement se reflète en syntaxe, ou même dans le langage? Autre point, la distinction entre adjectif comptable et adjectif de masse n'est pas clairement définie et ne repose que sur de vagues intuitions: en fait, les notions de Comptable et Masse sont vidées de leur contenu dans cette analyse et ne servent que d'étiquettes à une taxonomie de positions. Mumoratsu suggère qu'un adjectif de Taille comme small est comptable parce qu'il modifie une expression qui possède une forme. Mais les adjectifs de couleur sont des adjectifs de masse, et pourtant il semble bien qu'il faut avoir une forme pour avoir une couleur. D'autre part, le lien qui est fait entre être comptable et être 3D semble incorrect: comme l'a montré Vandeloise (1986), entre autres, les noms dénotant des objets concrets peuvent être de différentes dimensionalités: une file (1D), une plage (2D), ou une boîte (3D). Or les adjectifs dits comptables peuvent modifier chacun de ces types de noms.

(11) a. Ils ont formé une petite file devant le guichet.

b. Ils ont échoué sur une petite plage.

c. Elle a placé le vase dans une petite boîte.

La confusion vient de l'emploi trop relâché de la notion de dimensionalité. La suggestion que la Masse est 2D et l'Abstrait 1D ne fait aucun sens d'un point de vue cognitif et semble ne reposer que sur le désir de justifier différents niveaux syntaxiques. D'autre part, Mumoratsu propose la sériation en (12). 
La frontière entre $3 \mathrm{D}$ et $2 \mathrm{D}$ se situerait entre Forme et ÂGE, car, selon Mumoratsu, FORME fournit la troisième dimension (pourtant, les adjectifs de forme les plus courants sont 2D: carré, rond, allongé). De plus, TAILlE est plus élevé que FORME dans la hiérarchie car l'évaluation de la taille requiert une forme, dit-elle. Mais ceci n'est que vague dissertation. En fait, les principes de géométrie les plus élémentaires suggèrent le contraire: c'est la Forme qui exige une TAILlE dans une ou plusieurs dimensions (et non pas seulement en 3D). D'autre part, la sériation proposée en (12) est incorrecte sous plusieurs rapports, comme on le voit en (13):
a. He found an old cylindrical container in Bagdad.
[ÂGE $>$ FormE]
b. He found a red cylindrical container in Bagdad.
[COULEUR>FORME]
c. He found a Chinese cylindrical container in Bagdad. [Provenance>Forme]

En fait, l'analyse de Mumoratsu n'est pas vraiment cognitive mais syntaxique, les vagues correspondances avec des notions sémantico-cognitives ne servant qu'à justifier a posteriori des additions faites à la grammaire au niveau des catégories et enchâssements syntaxiques. En bout de compte, l'analyse nous dit très peu à propos de la sériation: il y a une liste ordonnée de positions, et on tente de motiver l'ordre sur la base d'une démarcation entre 3D et 2D, et d'un rapport entre TAILLE et Forme; mais nous avons vu que cela ne tient pas la route. De plus, de l'aveu même de Mumoratsu, l'analyse ne rend pas compte de l'ordre entre les adjectifs de même classe, comme ceux de masse dans old white ball/\#white old ball.

En général, les analyses sémantico-conceptuelles souffrent de deux faiblesses. D'une part, les notions utilisées sont souvent imprécises et relèvent trop d'intuitions simplistes, relevant de vagues impressions. D'autre part, on confond corrélation et explication: on constate qu'il y a un lien entre proximité du nom et adjectif exprimant une propriété inhérente/plus apparente/avec moins de calculs/de masse. Mais on n'indique pas pourquoi l'ordre des adjectifs devrait être sensible à ces facteurs. On n'indique pas non plus pourquoi c'est cette corrélation qui prévaut et non pas l'inverse, où l'adjectif à proximité du nom exprimerait une propriété non inhérente/moins apparente/ avec plus de calculs/comptable.

Mais ces faiblesses ne sont pas inhérentes à l'approche sémanticoconceptuelle: une suggestion faite par Ziff (1960) ouvre la voie à une analyse plus explicative, basée sur une notion plus précise.

\section{Modification adjectivale et formation de Concepts}

Comme nous l'avons vu dans la discussion de (5), les auteurs s'entendent généralement sur le fait que l'emboîtement syntaxique des adjectifs dépend de la portée sémantique des adjectifs: dans une suite d'adjectifs, 
qu'elle soit prénominale ou postnominale, l'adjectif dont la sémantique se prête le mieux à une modification directe du nom tend à se retrouver plus près de ce dernier. La question de fond est donc de déterminer en quel sens un adjectif modifie plus directement le nom qu'un autre adjectif. Ziff (1960 : 204-6) suggère d'abord que si deux adjectifs précèdent le nom, le premier a une plus grande classe d'environnements possibles. Ainsi, red précède wooden en (14a) car red s'applique à plus de noms que wooden, et il en va de même pour little qui précède white en (14b).
a. red wooden table
b. little white house

Toutefois, Ziff constate que le principe ne fonctionne pas dans plusieurs cas: il y a probablement plus d'hommes âgés que d'hommes intelligents, dit-il, et pourtant l'ordre usuel est celui en (15a); le principe échouerait aussi en (15b) selon Ziff.
a. intelligent old man
b. pious young girl

Il suggère que la notion de Sorte naturelle ('natural kind') est en jeu dans ces derniers exemples. Il n'est pas très explicite, mais on peut déduire de sa discussion que le nom et l'adjectif qui lui est adjacent décrivent une Sorte naturelle, qui est qualifiée plus avant par le second adjectif. Ceci semble être sur la bonne voie, car la notion de Sorte naturelle s'applique aussi à d'autres cas qui échappent aux analyses basées sur des notions comme le degré d'apparence ou la complexité de calculs. Deux cas se démarquent. Premièrement, Sproat \& Shih (1988) remarquent que les adjectifs de Provenance sont plus près du nom que les adjectifs de Taille (cela vaut aussi pour les adjectifs postnominaux du français):
a. enormous Chinese vase
\#Chinese enormous vase
b. vase chinois énorme
\#vase énorme chinois

Or on a l'impression qu'il faut des connaissances plus complexes pour établir qu'un vase est chinois que pour établir qu'il est énorme, ce qui contrevient à leur principe de sériation des adjectifs basé sur la complexité de calculs ${ }^{8}$. Selon Sproat \& Shih (1988), le placement des adjectifs de Provenance dépend d'un principe différent de celui qui rend compte du placement des adjectifs de Couleur, de Qualité, de Forme, de Taille, et ainsi de suite. Les adjectifs de Provenance servent typiquement d'indicateurs d'une taxonomie et on ne devrait pas être surpris, disent-ils, que ces adjectifs soient plus étroitement liés à la tête nominale que les autres adjectifs. Mais ils n'indiquent pas pourquoi il devrait être si évident qu'il y a une telle corrélation entre taxonomie et lien serré entre l'adjectif et le nom. 
En fait (et c'est là notre deuxième cas), la taxonomie a des effets qui s'étendent bien au-delà de la classe des adjectifs de Provenance: en contexte approprié, on peut aussi interpréter la plupart des adjectifs des autres classes comme établissant une taxonomie. Par exemple, alors qu'en (17a) on a l'ordre qui est usuel pour la plupart des contextes, on peut avoir l'ordre en (17b) si les petits chiens forment une classe pertinente dans le contexte de l'énoncé - si la Taille sert de base à une taxonomie - et que le locuteur veut référer à ceux qui sont bruns à l'intérieur de cette classe (voir aussi Whorf, 1945; Vendler, 1968; Dixon, 1982 : 24, et bien d'autres).

$$
\begin{aligned}
& \text { a. small brown dogs } \\
& \text { b. brown small dogs }
\end{aligned}
$$

On note donc que la sériation des adjectifs n'a pas la rigidité prédite par nombre d'analyses. D'autre part, les cas de taxonomie comme en (16) et (17) ne s'expliquent pas par le degré d'apparence ou la complexité des calculs. Par contre, la notion de Sorte naturelle permet une analyse qui rend compte non seulement des adjectifs naturellement taxonomiques comme ceux de Provenance, et des adjectifs contextuellement taxonomiques comme en (17b), mais qui se généralise à tous les cas d'emboîtement des adjectifs.

Précisons d'abord la notion de Sorte naturelle. Comme l'indique Chierchia (1998) (s'appuyant sur Carlson 1980 : 26sv. et Krifka et al. 1995), d'un point de vue intuitif, les Sortes naturelles sont des régularités qu'on retrouve dans la nature. Ce qui compte comme étant une Sorte naturelle n'est pas établi par la grammaire, mais par les connaissances que partage une communauté de locuteurs. Plusieurs classes comprennent des objets qui ont une fonction ou un comportement suffisamment réguliers pour se qualifier invariablement comme des Sortes naturelles. Il s'agit là de cas typiques. Toutefois, si le contexte s'y prête, d'innombrables classes peuvent être considérées comme suffisamment stables pour que le locuteur les présente à son interlocuteur comme formant une Sorte. Ces classes peuvent être construites dans la langue par une description, comme dans des noms complexes comme musique forte, citron sucré, célibataire endurci. Ces noms complexes peuvent même dénoter des classes présentées par le locuteur comme étant suffisamment bien établies pour qu'ils puissent apparaître dans un environnement généralement réservé aux Sortes, comme le notent Krifka et al. (1995 : 112). Par exemple, (18) est acceptable dans un contexte où les bouteilles vertes ne sont pas simplement des bouteilles qui se trouvent à être vertes, mais sont des objets qui ont une propriété additionnelle qui est stable dans le contexte et qui provient de la combinaison d'être vert et d'être une bouteille, comme dans le cas où les bouteilles vertes permettraient à un certain médicament de mieux se conserver: 
The green bottle saved the lives of hundreds of children.

La stabilité de la classe peut être très relative, de courte durée, le concept étant construit contextuellement. Cette façon de procéder est très productive dans les langues. Ainsi, en chinois, certains classifieurs comme wèi ont une portée sur des expressions nominales exprimant une Sorte. Or, comme le note Krifka (1995 : 402), des expressions nominales modifiées par des adjectifs ou des phrases relatives peuvent être sous la portée de wèi. Par exemple, le modifieur chuan lán yifu de est sous la portée de wèi en (19), de sorte que non seulement xiansheng 'monsieur' réfère à une Sorte, mais aussi chuan lán yifu de xiansheng 'monsieur, qui porte des vêtements bleus'.

$$
\begin{aligned}
& \text { nèi wèi [chuan lán yifu de] xiansheng } \\
& \text { que CL porte bleu tissu SUB monsieur } \\
& \text { 'ce monsieur, qui porte des vêtements bleus' }
\end{aligned}
$$

Krifka suggère d'utiliser le terme de 'Concept' pour distinguer ces cas plus contextualisés et productifs des cas de Sorte:

One possible analysis is to introduce a notion that is more general than that of a kind. So far, kinds were considered to be abstract entities that are well established in the background knowledge of speaker and hearer and can be referred to by definite NPs like the bear, which were in the extension of kind predicates like be extinct or be a mammal, and which were organized in taxonomic hierarchies. Let us now assume a new type of entities, concepts. Similar to kinds, concepts are abstract entities related to real objects. However, they need not be well established, but could be construed from scratch.

Cette notion de Concept construit contextuellement permet de rendre compte de la sériation des adjectifs, de préciser l'observation générale que l'adjectif dont la sémantique se prête le mieux à une modification directe du nom tend à se retrouver plus près de ce dernier. Essentiellement, dans un certain contexte, l'adjectif perçu comme étant le plus susceptible de former un concept avec le nom est celui qui se combine immédiatement avec le nom, qui est le plus enchâssé. Dans une solution européenne commune (6a), en juxtaposant européenne à solution, le locuteur les présente à son interlocuteur comme formant un concept. Si certains considèrent cet ordre comme plus naturel que celui dans une solution commune européenne (6b), c'est parce qu'il leur est plus facile d'imaginer un contexte où solution européenne forme une classe stable que l'on qualifie de commune, plutôt que solution commune que l'on qualifie d'européenne. Mais il ne s'agit là que de considérations strictement contextuelles et non grammaticales. C'est pourquoi un changement de contexte peut permettre des variations dans ce qui est présenté comme formant un concept, entraînant ainsi des changements dans la sériation des adjectifs. Certains emboîtements d'adjectifs sont beaucoup plus 
rigides, comme red wooden table (14a): c'est qu'il est généralement plus pertinent pour des motifs cognitifs d'identifier la classe des tables en bois que celle des tables rouges. Mais un contexte particulier pourrait faire ressortir le concept de tables rouges comme étant plus pertinent et nous amener à renverser cette tendance, et donc à inverser l'ordre des adjectifs: la couleur servirait alors à établir une taxonomie ${ }^{9}$. Certains adjectifs, comme ceux de Provenance, expriment une propriété qui est souvent utile pour identifier les éléments: ils ont donc tendance à être directement combinés au nom parce qu'il sont fortement susceptibles de former un concept avec le nom, une classe stable. Il n'est pas étonnant qu'on les désigne comme étant taxonomiques: une taxonomie est une classification sur la base de propriétés communes suffisamment stables pour être utiles dans l'identification des éléments. On comprend maintenant les exemples qui créaient un problème pour le premier principe de Ziff: l'adjectif avec la plus petite classe d'environnements possibles n'est pas nécessairement celui qui est le plus régulièrement pertinent pour identifier une classe. Ainsi, il est plus régulièrement pertinent d'identifier la classe des hommes âgés que celle des hommes intelligents, d'où (15a), et la classe des filles jeunes que des filles pieuses, d'où (15b).

On peut donc énoncer le principe général suivant:

Principe général de sériation des adjectifs:

Plus la propriété exprimée par un adjectif permet de former avec le nom un Concept pertinent et usuel, plus cet adjectif a tendance à être près du nom, c'est-à-dire, à modifier plus directement le nom qu'un autre adjectif.

Ce principe s'inscrit dans le contexte très large de la propension qu'ont les humains à créer des Concepts, tantôt permanents (ils ont alors une entrée dans le lexique mental), tantôt provisoires (ils sont alors construits contextuellement au besoin, comme dans des combinaisons de nom et d'adjectif). Ce principe général prédit une rigidité toute relative de l'ordre des adjectifs, et on pourrait lui reprocher d'être trop permissif et dépendant du contexte. Mais ce principe est en accord avec les faits: la rigidité apparente de la sériation des adjectifs ne provient que d'une limitation sans fondement des contextes pris en compte. La langue considérée dans son ensemble exhibe des variations dans le placement des adjectifs, et une théorie linguistique qui n'en rend pas compte est erronée. Il y a une tendance forte pour un certain ordre des adjectifs, mais ce n'est qu'une tendance, et elle s'explique par la vraisemblance relative des Concepts qui sont construits. Les exemples qui suivent présentent quelques paires d'adjectifs de diverses classes et montrent bien à quel point la sériation dépend d'un principe comportant une certaine relativité contextuelle comme (20). 
En général, l'ordre de composition des adjectifs dépend de nos attentes en fonction de nos connaissances. Par exemple, comme l'ont noté Sproat \& Shih (1988), les adjectifs de Provenance servent typiquement d'indicateurs d'une taxonomie, donc ont tendance à se construire directement avec le nom comme en (8) ci-dessus. Mais dans un contexte où l'on discute des différentes techniques et traditions pour obtenir du bleu en poterie, la couleur devient plus pertinente, et on obtient naturellement l'ordre en (21).

a. vases bleus chinois

b. Chinese blue vases

Les adjectifs de couleur expriment une propriété qui permet très souvent de distinguer entre les objets. Ils ont donc fortement tendance à former directement un Concept avec le nom. Mais selon le nom et l'autre adjectif que l'on choisit, la pertinence du Concept classificateur peut varier. Ainsi, la couleur et la taille qu'expriment les adjectifs en (22) peuvent toutes deux facilement être pertinentes pour une amibe, d'où les deux ordres (avec des différences de sens): on peut soit avoir le Concept d'amibes transparentes parmi lesquelles ont fait ressortir celles qui sont microscopiques (22a), ou l'inverse (22b).

(22) a. amibes transparentes microscopiques

a'. microscopic transparent amebas

b. amibes microscopiques transparentes

b'. transparent microscopic amebas

De même, on serait (vainement) à la recherche de contenants cylindriques en (23a), alors que ce sont les contenants rouges qui sont plus pertinents en (23b), tandis qu'en (24a), je pourrais avoir trouvé plusieurs pianos noirs, mais aucun qui soit antique, mais l'inverse en (24b).

a. They did not find any red cylindrical containers in Bagdad. Ils n'ont pas trouvé de contenants cylindriques rouges Bagdad.

b. They did not find any cylindrical red containers in Bagdad. Ils n'ont pas trouvé de contenants rouges cylindriques à Bagdad.

(24) a. Je cherche un piano noir antique.

I am looking for an antique black piano.

b. Je cherche un piano antique noir.

I am looking for a black antique piano.

Le placement des adjectifs évaluatifs comme bon, beau, horrible est beaucoup plus rigide: comme on le voit en (25), il leur est difficile de former directement un Concept avec le nom.

a. une bonne grosse maison; a good large house; \#une grosse bonne maison

\#a large good house 
$\begin{array}{ll}\text { b. un beau petit chien; } & \text { \#un petit beau chien } \\ \text { a nice small dog; } & \text { \#a small nice dog }\end{array}$

Ces adjectifs sont éloignés du nom dans la sériation parce que la propriété qu'ils expriment est fort subjective, et elle sert donc difficilement de base à une taxonomie puisqu'elle est peu utile pour distinguer entre des éléments.

\section{Conclusion}

La sériation des adjectifs est très semblable d'une langue à l'autre, non pas en terme d'ordre absolu, mais plutôt de distance relativement au nom. Cette sériation n'est pas absolument rigide, mais apparaît comme une tendance qui est reliée à la propension qu'ont les humains à créer des Concepts. L'adjectif le plus près du nom est celui qui permet le plus naturellement de construire un Concept avec le nom. Le Concept peut être très contextualisé et provisoire. C'est ce qu'exprime le principe en (20). L'embồtement des adjectifs dans la sériation correspond à un emboîtement de Concepts, de classes que l'on forme: plus la classe est naturelle, plus immédiatement l'adjectif est combiné avec le nom. Quand la tendance pour une sériation est très forte, comme Provenance qui est plus près du nom que Taille, mon analyse dit que c'est parce qu'il est plus naturel pour le système cognitif des humains de former la classe Provenance + Nom que la classe Taille + Nom. Il reste à vérifier s'il y a vraiment une telle hiérarchie quant au naturel des Concepts, et sur quelle base elle repose. Mais cela dépasse le cadre d'une étude en linguistique. Le présent travail a au moins le mérite de permettre qu'on pose la question avec assez de précision pour l'aborder ultérieurement.

\section{NOTES}

1. Je remercie les deux évaluateurs anonymes de Recherches linguistiques de Vincennes pour leurs commentaires et suggestions.

2. Voir aussi Blinkenberg (1933: 128). On retrouve cette observation chez presque tous les linguistes qui ont étudié cette construction.

3. La notion de position naturelle d'interprétation est basée sur des intuitions naïves provenant d'observations désinvoltes, qui provoquent plusieurs problèmes fondamentaux et sont la raison pour laquelle les analyses en grammaire générative ne sont souvent en bout de compte que des listes qui ne font que répéter des descriptions superficielles des faits. Voir Bouchard (2005) pour une critique générale et une proposition de solution.

4. L'ajout de nombreuses catégories syntaxiques correspondant à des classes sémantico-conceptuelles n'est pas sans rappeler la mésaventure de la sémantique générative. Comme je le souligne dans Bouchard (2002 : 336), le résultat est le même, c'est-à-dire un système dont chaque étape de la dérivation semble contrainte, mais qui 
ne l'est pas dans son ensemble quand on prend en compte tout ce que permettent les opérations et leurs déclencheurs.

5. La distinction entre propriété absolue et propriété relative a été utilisée depuis Aristote pour caractériser les classes d'adjectifs.

6. Bunt et Mumoratsu utilisent le symbole d'agrammaticalité (*) ici, mais j'emploie plutôt le symbole marquant l'anomalie sémantico-pragmatique (\#).

7. Pour rendre compte de l'ordre miroir des adjectifs postnominaux dans les langues romanes, Mumoratsu propose une variation de l'analyse de Cinque (1994) comme pour $(8 b)$ ci-dessus.

8. Je n'admets cette impression de Sproat et Shih que pour les fins de l'argument: aucune base scientifique n'est fournie pour étayer cette affirmation.

9. Les exemples en (17) montrent une inversion semblable, mais pour les classes de Taille et Couleur. Plusieurs des auteurs cités à cet exemple notent qu'il y a souvent une pause et/ou une intonation contrastive quand on ne suit pas l'ordre typique. Je crois que ces marques servent à alerter en quelque sorte l'interlocuteur qu'il s'agit d'une forme exceptionnelle, non pas du point de vue de la syntaxe, mais dans le sens qu'il s'agit d'une classe naturelle inhabituelle. Sur le jeu des intonations dans l'interprétation des adjectifs, voir Bouchard (2002 : 188sv).

\section{RÉFÉRENCES BIBLIOGRAPHIQUES}

ABney, Steven (1987). The English Noun Phrase in its Sentential Aspect. Thèse de doctorat. Cambridge MA: Massachusetts Institute of Technology.

Bernstein, Judy (1993). Topics in the Syntax of Nominal Structure across Romance. Thèse de doctorat. New York: City University of New York.

Blinkenberg, Andreas (1933). L'Ordre des mots en français moderne: Deuxième partie. Copenhague: Levin and Munksgaard.

BouchaRD, Denis (2002). Adjectives, Number and Interfaces: Why Languages Vary. Oxford: Elsevier Science.

BouchARD, Denis (2005). Exaption and Linguistic Explanation. Lingua 115:16851696.

Bunt, H.C. (1979). Ensembles and the Formal Semantic Properties of Mass Terms. Dans Pelletier, F.J. (ed.), Mass Terms : Some Philosophical Problems: 249277. Dordrecht: D. Reidel.

CARlson, Gregory (1980). Reference to Kinds in English. New York: Garland Publishing.

Chierchia, Gennaro (1998). Reference to kinds across languages. Natural Language Semantics 6: 339-405.

Chomsky, Noam (2000). Minimalist inquiries: The framework. Dans Martin, R.; Michaels, D.; Uriagereka, J. (eds.), Step by step : Essays on minimalist syntax in honor of Howard Lasnik: 89-155. Cambridge MA: MIT Press.

Cinque, Guglielmo (1994). On the Evidence for Partial N Movement in the Romance DP. Dans Cinque, G.; Koster, J.; Pollock, J.-Y.; Rizzi, L. \& Zanuttini, R. (eds.), Paths Towards Universal Grammar: 85-110. Georgetown: Georgetown University Press.

Cinque, Guglielmo (1999). Adverbs and Functional Heads. Oxford: OUP. 
Dixon, Robert (1982). Where Have All the Adjectives Gone? La Haye: Mouton.

Forsgren, Mats (1978). La Place de l'adjectif épithète en français contemporain; Étude quantitative et sémantique. Stockholm: Almqvist \& Wiskell.

Grevisse, Maurice (1986) [1936]. Le Bon Usage. Gembloux: Duculot.

KrIFKA, Manfred (1995). Common Nouns: a contrastive analysis of Chinese and English. Dans Carlson, G. \& Pelletier, F.J. (eds.), The Generic Book: 398-411. Chicago: The University of Chicago Press.

KrifKa, Manfred; Pelletier, Francis Jeffry; Carlson, Gregory N.; TER Meulen, Alice; Link, Godehard; ChIERChIA, Gennaro (1995). Genericity: An introduction. Dans Carlson, G. \& Pelletier, F.J. (eds.), The Generic Book: 1124. Chicago: The University of Chicago Press.

LEECH, Geoffrey Neil; SVARTVIK, Jan (1975). A Communicative Grammar of English. London: Longman.

MARTin, J.E. (1969a). Some competence-processing relationships in noun phrases with prenominal and postnominal adjectives. Journal of Verbal Learning and Verbal Behavior 8: 471-480.

Martin, J.E. (1969b). Semantic determinants of preferred adjective order. Journal of Verbal Learning and Verbal Behavior 8: 697-704.

Mumoratsu, Keiko (2001). Adjective ordering as the reflection of a hierarchy in the noun system. Dans Pica, P. \& Rooryck, J. (eds.), Linguistic Variation Yearbook 1: 181-207. Amsterdam/Philadelphia: John Benjamins Publishing.

SPROAT, Richard; SHIH, Chilin (1988). Prenominal Adjectival Ordering in English and Mandarin. Proceedings of NELS 18: 465-489.

VALOIS, Daniel (1990). The internal syntax of DP and adjective placement in English and French. Proceedings of NELS $21: 367-381$.

VALoIs, Daniel (1991). The Internal Syntax of DP. Thèse de doctorat. Los Angeles, CA: UCLA.

VANDERLOISE, Claude (1986). L'Espace en français. Paris : Éditions du Seuil.

VENDLER, Zeno (1968). Adjectives and Nominalizations. La Haye: Mouton.

WHORF, Benjamin (1945). Grammatical categories. Language 21 : 1-11.

ZIFF, Paul (1960). Semantic analysis. Ithaca: Cornell University Press. 


\begin{abstract}
In French, there is a preferred order for adjectives when they are on the same side of the Noun inside a noun phrase. These adjectives are interpreted as being stacked: the adjective immediately juxtaposed to the noun modifies this noun, the adjective juxtaposed to this constituent modifies it, and so on. The seriations depend on the meaning of the adjectives and the context of use: the adjective most likely to form a concept with the noun given the situation of use is the one which immediately combines with the noun, which is the most deeply embedded. The seriations only indicate preferences and are not rigid constraints, and it is almost always possible to invert the order of the adjectives. The resulting combinations are not syntactically deviant, but rather are less expected semantic combinations: the expressions are acceptable if the noun and adjective form a concept which is appropriate in the context.
\end{abstract}

\title{
KEYWORDS
}

Adjectives, order, temporary concepts. 